Communications in Physics, Vol.26, No. 1 (2016), pp. 93-97

DOI: $10.15625 / 0868-3166 / 26 / 1 / 7474$

\title{
A LOW BACKGROUND GAMMA RAY SPECTROMETER WITH ANTICOSMIC SHIELDING
}

\author{
NGUYEN QUOC HUNG ${ }^{\dagger}$, VO HONG HAI, TRAN KIM TUYET, AND HO LAI TUAN \\ University of Science, HCMC-Vietnam National University, \\ 227 Nguyen Van Cu, Ward 4, District 5, Ho Chi Minh City, Vietnam \\ ${ }^{\dagger}$ E-mail: nqhung@hcmus.edu.vn \\ Received 27 November 2015 \\ Accepted for publication 14 April 2016
}

\begin{abstract}
The article describes a gamma ray spectrometer protected by a lead shield (Model $747 E$ Canberra lead shield) and an active shield made of an $80 \mathrm{~cm} \times 80 \mathrm{~cm} \times 3 \mathrm{~cm}$ plastic scintillator plate in anticoincidence on top of the lead shield. The detector used as low background gamma-spectrometer is a high purity Germanium crystal of model GC2018 Canberra. The background count rate currently achieved (30-2400 keV) is $1.27 \mathrm{cps}$ without anticoincidence. The level of background suppression obtained from the active protection is 0.80 overall and about 0.43 for the $511 \mathrm{keV}$ gamma line. The gamma ray spectrometer is installed and operated in the Nuclear Laboratory, Department of Nuclear Physics, University of Science, HCMC-Vietnam National University.
\end{abstract}

Keywords: gamma ray spectrometer, cosmic rays, plastic scintillator, high-purity Germanium detector.

Classification numbers: $29.30 . K v, 96.50 . S$ -

\section{INTRODUCTION}

The background of a Germanium detector is due to natural and artificial radioactive elements distributed in the surroundings of the detector, radon and cosmic rays induced radioactivity [1]. The major sources of activity from the crystal and the construction materials are primordial emitters ${ }^{238} \mathrm{U},{ }^{232} \mathrm{Th}$ and their daughters, and ${ }^{40} \mathrm{~K}$ isotope in natural potassium. This contribution to the background can be reduced by means of suitable shielding (very low activity lead or "old" lead) and by selecting pure radioactivity materials surrounding the crystal [2]. The radon component can be reduced by flushing the cavity around a detector with nitrogen evaporating from the Dewar [3]. Then the cosmic rays induced background become predominant, which originates from cosmic muon and the production of tertiary neutron by muon in the surrounding materials,

(c)2016 Vietnam Academy of Science and Technology 
especially the lead shield $[4,5]$. This component can be reduced by installing the Ge-detector in an underground laboratory [6-8]. The other possibility is to operate a Germanium detector by an anticosmic shielding [8]. The anticosmic shield can be made of gas or plastic scintillation detectors, which surround the lead shield housing the Ge-detector [1,9]. In this work, we have designed and implemented a background reduction system for a Ge-detector located at a surface laboratory with plastic scintillation plates and an anticoincidence electronic system.

\section{DETECTOR SYSTEM}

The Ge-detector used in this work is a low-level high-purity Ge-detector (HPGe), made by Canberra (model GC2018), with a relative efficiency of $20 \%$, an active volume of $104 \mathrm{~cm}^{3}$ and an energy resolution of $1.8 \mathrm{keV}$ (full width at half maximum, FWHM) at $1332.5 \mathrm{keV}$ gamma ray of ${ }^{60} \mathrm{Co}$ [10]. The Ge-detector is installed in the surface laboratory of the Department of Nuclear Physics, Faculty of Physics \& Engineering Physics, University of Science, VNU-HCMC.

\section{Passive shield}

The whole laboratory is located above ground in a building room of the department with 20 $\mathrm{cm}$ thick concrete walls as a first layer of shielding. The detector is surrounded by a cylindrical bulk shield (Model 747E made by Canberra) $63.5 \mathrm{~cm}$ high with a diameter of $50.8 \mathrm{~cm}$ [11]. It consists of the following shielding layers (moving inward): $9.5 \mathrm{~mm}$ thick low carbon steel, $10 \mathrm{~cm}$ thick low-background lead, $1.0 \mathrm{~mm}$ tin and $1.6 \mathrm{~mm}$ copper graded liners to reduce or eliminate the presence of characteristic fluorescence X-rays from the lead shield. The whole detector assembly is placed inside a lead shield, $10 \mathrm{~cm}$ below its upper surface.

\section{Anticoincidence setup}

An active shielding consisting of two $80 \times 40 \times 3 \mathrm{~cm}^{3}$ plastic scintillator plates placed side by side, equivalent to an $80 \times 80 \times 3 \mathrm{~cm}^{3}$ plastic veto scintillator, is located above the upper side of the passive shielding. The single rate in the scintillator plates is approximately $70 \mathrm{cps}$ (counts per second). The signal from the scintillator plate is subjected to a $25 \mathrm{mV}$ discriminator threshold, equivalent to the $2.0 \mathrm{MeV}$ energy deposition for muons and gamma rays. The main aim of the active shield is to eliminate the cosmic-ray background (mainly induced by cosmic muons). This background consists of cosmic particles (essentially muons, but also neutrons) which may interact or decay in the material between the anticoincidence counter and the germanium detector, producing a background not only of decay electrons but also of delta rays and soft gamma rays. To reject it, in order to allow for the important contribution of electrons from muon decays, one needs therefore to allow for a time delay reaching of the order of the muon lifetime ( $2.2 \mu \mathrm{s})$ between the fast scintillator pulse and the germanium signal.

The electronics diagram is displayed in Fig. 1. NIM electronics is used to generate the veto gate. The scintillator pulses are amplified, converted to fast logic signals using constant fraction discriminators (CFD) and OR-ed; the OR signal is then processed in a delay/gate generator, $2.2 \mu \mathrm{s}$ wide and $200 \mathrm{~ns}$ delayed. The Ge-pulse is processed by a preamplifier (Canberra 2002C) and an amplifier (Canberra 2026), then input to the "ADC INPUT" of MCA-EASY-2K (MCA made by ORTEC). Besides, the amplifier has an ICR (incoming count rate) output which provides a standard TTL logic signal corresponding to the input count rate. The digital signals of the Ge-detector 


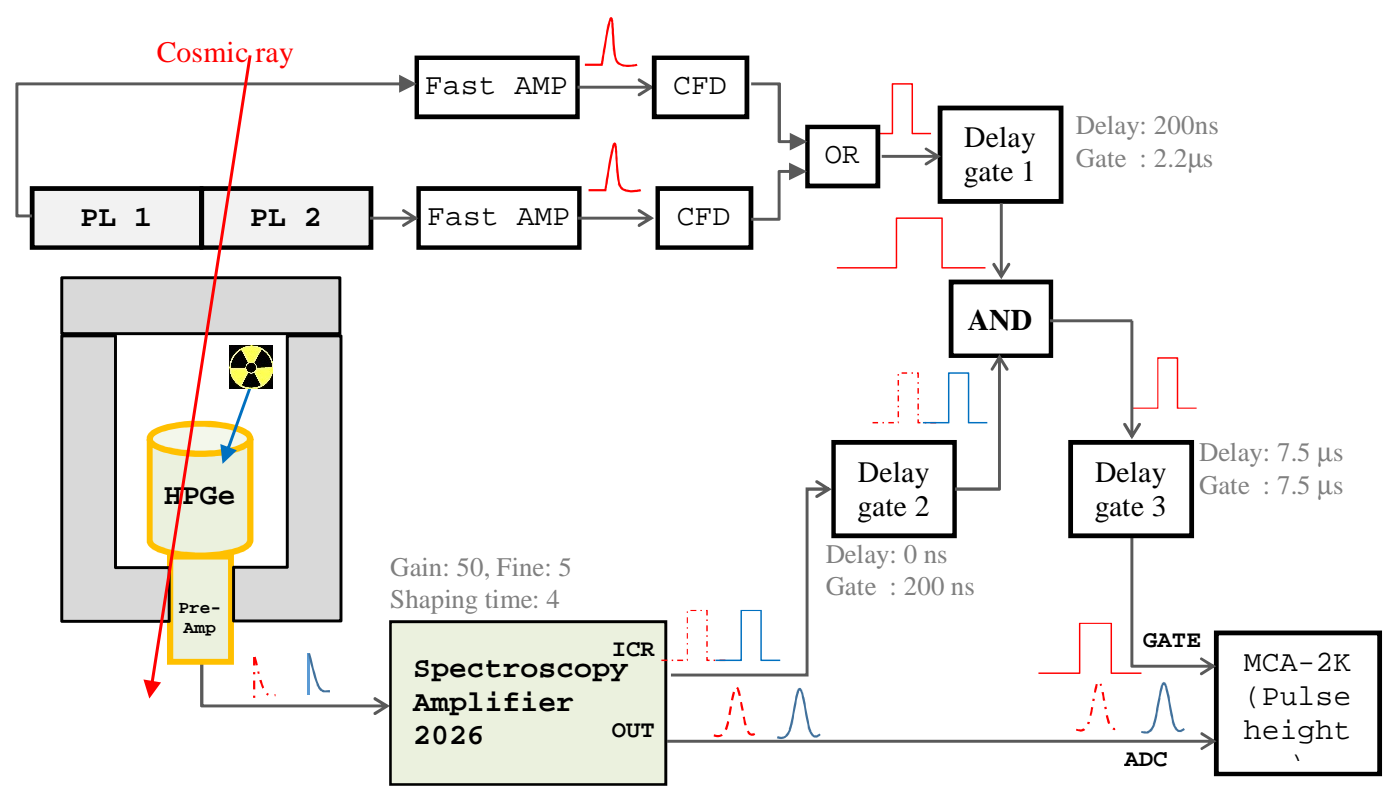

Fig. 1. Schematic diagram of the electronics.

and the plastic scintillator veto gate are then AND-ed. As a result, an output "gate signal" of the active veto shield is produced only when there is a coincidence signal between the plastic scintillator detector and the Ge-detector and then sent directly to the gate input on the multichannel analyzer MCA-EASY-2K. The MCA is software-selectable to record detector pulses in coincidence, anticoincidence or "off" with regard to this gate signal. The gate signal from the AND-combination has to be delayed by $7.5 \mu \mathrm{s}$ and extended to $7.5 \mu \mathrm{s}$ by a second delay/gate generator to symmetrically overlap the FLAT TOP section of the shaped signal from the spectroscopy amplifier. In this work, the anticoincidence mode is selected, in order to eliminate the Ge-detector pulses produced by cosmic rays. The delays, width extensions (delay/gate generators), OR-combination and ANDcombination are done with the FPGA trigger module which is embedded by VHSIC Hardware Description Language (VHDL) code for Field-Programmable Gate Array (FPGA) technology.

\section{RESULTS AND DISCUSSION}

Fig. 2 shows a comparison of the background without and with veto detector. Integrated counting rates between $30 \mathrm{keV}$ and $2400 \mathrm{keV}$ are $1.27 \mathrm{cps}$ and $1.02 \mathrm{cps}$; respectively. This means that the present active shield itself reduces the background by a factor of 1.23 . When a muon collides with high $\mathrm{Z}$ materials, it produces many kinds of secondary particles such as electrons, positrons and protons. These secondary particles are either absorbed by lead shielding or generate gamma rays by bremsstrahlung or electron-positron annihilation. The $511 \mathrm{keV}$ gamma line shown in Fig. 2 is mainly due to annihilations induced by cosmic bremsstrahlung positrons. The active shield, suppresses it by a factor of 2.29. The reduction in various energy intervals in the spectrum is broken down in Table 1. The effectiveness of the veto is not as strong below $100 \mathrm{keV}$, where 
the reduction factor falls quickly to around a factor of 1.1 . Above $600 \mathrm{keV}$, the overall reduction is around a factor of 1.3. The background reduction that has been obtained is small and would remain small if a better anticoincidence counter, with broader solid angle coverage were used. The main source of background is due to ambient radon and neutrons, which do not interact in the anticoincidence shield.

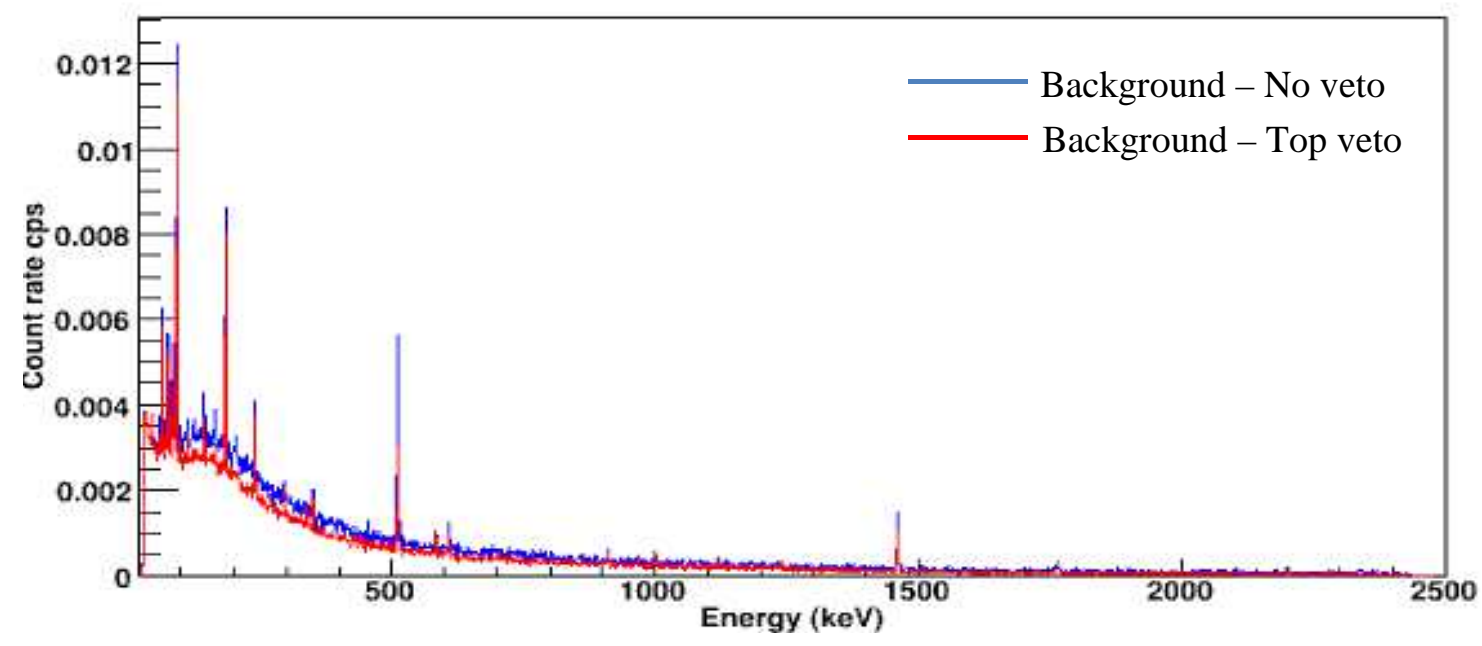

Fig. 2. Comparing the background spectra obtained without and with active shield.

Table 1. Background reduction in counting rates across various energy intervals, without and with active shielding.

\begin{tabular}{llll}
\hline $\begin{array}{l}\text { Region } \\
(\mathrm{keV})\end{array}$ & $\begin{array}{l}\text { No veto } \\
\text { (c.p.s) }\end{array}$ & $\begin{array}{l}\text { Top veto } \\
\text { (c.p.s) }\end{array}$ & Reduction \\
\hline $30-50$ & 0.054 & 0.053 & 1.019 \\
$50-100$ & 0.168 & 0.149 & 1.128 \\
$100-150$ & 0.143 & 0.121 & 1.187 \\
$150-200$ & 0.139 & 0.117 & 1.195 \\
$200-250$ & 0.115 & 0.092 & 1.248 \\
$250-300$ & 0.085 & 0.068 & 1.252 \\
$300-400$ & 0.123 & 0.097 & 1.268 \\
$400-500$ & 0.081 & 0.064 & 1.269 \\
511 & 0.021 & 0.009 & 2.287 \\
$500-600$ & 0.073 & 0.054 & 1.366 \\
$600-2400$ & 0.310 & 0.225 & 1.376 \\
$30-2400$ & 1.273 & 1.023 & 1.244 \\
\hline
\end{tabular}




\section{ACKNOWLEDGEMENTS}

The authors would like to express their sincere thanks to RCNP, Osaka University, Japan in supporting the FPGA trigger module; to Prof. Chary Rangacharyulu, University of Saskatchewan, Canada in supporting the multichannel analyzer MCA-EASY-2K; and to the Nuclear Technique Laboratory, University of Science, VNU-HCM for providing several NIM modules. We also thank Prof. Pierre Darriulat and his VATLY group for providing the large plastic scintillator plates. This work was supported by University of Science, HCMC-Vietnam National University, under grant number T2015-04.

\section{REFERENCES}

[1] G. Heusser, Nuclear Instruments and Methods in Physics Research Section B: Beam Interactions with Materials and Atoms 17 (5) (1986) 418-422.

[2] P. Vojtyla, Nuclear Instruments and Methods in Physics Research Section B: Beam Interactions with Materials and Atoms 111 (1) (1996) 163-170.

[3] H. Malm, M. Watt, I. Bostock, J. Campbell, P. Jagam, and J. Simpson, Nuclear Instruments and Methods in Physics Research 223 (2-3) (1984) 420-425.

[4] W. Preusse and S. Unterricker, Nuclear Instruments and Methods in Physics Research Section B: Beam Interactions with Materials and Atoms 94 (4) (1994) 569-574.

[5] D. Haines, T. Semkow, A. Khan, T. Hoffman, S. Meyer, and S. Beach, Nuclear Instruments and Methods in Physics Research Section A: Accelerators, Spectrometers, Detectors and Associated Equipment 652 (1) (2011) 326-329.

[6] R. M. Lindstrom, D. J. Lindstrom, L. A. Slaback, and J. K. Langland, Nuclear Instruments and Methods in Physics Research Section A: Accelerators, Spectrometers, Detectors and Associated Equipment 299 (1-3) (1990) 425-429.

[7] P. Finnerty, S. MacMullin, H. Back, R. Henning, A. Long, K. Macon, J. Strain, R. Lindstrom, and R. Vogelaar, Nuclear Instruments and Methods in Physics Research Section A: Accelerators, Spectrometers, Detectors and Associated Equipment 642 (1) (2011) 65-69.

[8] K. Thomas, E. Norman, A. Smith, and Y. Chan, Nuclear Instruments and Methods in Physics Research Section A: Accelerators, Spectrometers, Detectors and Associated Equipment 724 (2013) 47-53.

[9] T. Schroettner, M. Schwaiger, and P. Kindl, Applied radiation and isotopes 61 (2) (2004) 133-138.

[10] Germanium Detectors, Users Manual, https://www1.aps.anl.gov/files/download/DET/ Detector-Pool/Spectroscopic-Detectors/Canberra/Germanium_Detectors_Manual.pdf.

[11] Detector Shields, Model 747E Lead Shield, http://www.canberra.com/products/detectors/ detector-shields.asp. 